\title{
LXXI. Theory of the spirit-level
}

\section{J. Nixon Esq.}

To cite this article: J. Nixon Esq. (1827) LXXI. Theory of the spirit-level, Philosophical Magazine Series 2, 1:5, 354-364, DOI: 10.1080/14786442708674329

To link to this article: http://dx.doi.org/10.1080/14786442708674329

曲 Published online: 10 Jul 2009.

Submit your article to this journal 중

Џ Article views: 2

Q View related articles $\sqsubset$ 
Vlacq's great work was printed at Gouda in 1628 : and upon examination I find the following results for the numbers in Mr. Babbage's list.

\begin{tabular}{|c|c|c|}
\hline & $\begin{array}{l}\text { Logarithms as printed } \\
\text { by Vlacq. }\end{array}$ & $\begin{array}{l}\text { Logarithms as they ought } \\
\text { to have been printed. }\end{array}$ \\
\hline 24626 & ... 39139.39751. & . . $39139 \cdot 3 \overline{8} 751$ \\
\hline 38962 & . . . 59064.134.20 & . $59064 \cdot 1 \overline{2} 420$ \\
\hline 57628 & $76063 \cdot 35875$ & $76063 \cdot 35 \overline{4} 75$ \\
\hline 57629 & . $76064 \cdot 10436$ & $76064 \cdot 10 \overline{8} 36$ \\
\hline 63747 & .. $80445^{\circ} 974,12$ & $80445 \cdot 97 \overline{5} 12$ \\
\hline 67951 & $\ldots .83219 \cdot 58424$ & . $83219 \cdot 58 \overline{5} 24$ \\
\hline
\end{tabular}

I have placed a mark over the corrected figures : and it will be clear that, if we reserve only seven places of decimals, - and we wish to have the last of them true to the nearest figure,Mr. B.'s corrections will be necessary for the last five numbers in this list. It should, however, be remarked in justice to Taylor, that he had detected the mistake in the logarithm of 38962, and has noted, in the errata printed on the last page of his tables, that the last figures should be 412, not 413. Indeed the cost of time, labour and attention must have been immense, to have avoided a greater number of errors. Some idea of the difficulty may be formed, when it is seen that even Mr. Babbage, with all his most landable anxiety for accuracy, has not been able to avoid mistake. It seems to have escaped him that Vlacq's logarithms for 24626 was wrong, and that the last figures of the seven decimals are, therefore, not 940 , as he suggests that they ought to be, and as they are in Gara diner and Hutton, but 939 , as they are given by Taylor.

Allow me to take this opportunity of pointing out to you that your note at page 271 is incomplete. You should have added that M. Gay-Lussac, who ascended higher with his balloon than MM. Sacharoff and Robertson, found no such variation as they describe, in the intensity of the earth's magnetism. There is an account of his voyage in the $21 \mathrm{st}$ volume of the Philosophical Magazine, at page 220.

April 12, 1827.

N. R. D.

LXXI. Theory of the Spirit-Level. By J. Nixon, Esq.

[Concluded from p. 263.]

TN order to acquire some knowledge of the variation of figure produced in the bubble of a spirit-level in consequence of the reciprocal attraction of the glass tube and the 
contained liquid, the following experiments were made with a straight glass tube having a bore of 0.5 inch. Both ends being stoppered, an opening $a$, of an irregular figure, about 0.2 in. across and $0.3 \mathrm{in}$. deep, was made in a part of the tube equidistant from the ends. The tube being placed horizontally with the orifice upwards, was filled with water, which stood within it
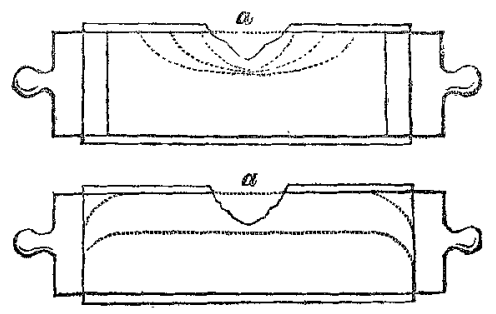
apparently the same in volume and figure as though the tube had been entire and impervious to the air. Drawing out the stoppers, so as gradually to augment the space between them, the atmospheric air soon made its ingress, causing that portion of the water immediately under the orifice on which it was incumbent to assume the concave surface, of which a section is given in the upper figure. But as the interior space continued to augment, the bubble of air elongated towards the stoppers without material addition to its depth; its ends being equidistant from $a$, and curved precisely as those of the bubble of a spirit-level. On pushing the stoppers home, the bubble, repassing through the same changes of figure, was finally expelled from the tube.

The tube being well dried, the experiment was repeated with mercury, which not only filled the tube, but stood at $a$ quite out of it. On increasing the interior space, the mutual repulsion of glass and mercury gave room to the latter to shrink in the first instance from the upper corners of the tube, thus checking the admission of the air until a further addition of space within the tube caused the mercury to subside throughout its length with a surface nearly horizontal, but terminating at the stoppers in a convex curve as before*.

In order to enable us to comprehend, in some degree, why the water filling the horizontal tube, so soon as the containing space became enlarged, should not subside equally the whole length of the tube, and come to rest with a horizontal surface of which the bounding lines (a right-angled parallelogram) would be in every point in contact with the sides of the tube or ends of the stoppers, it will be necessary to notice such of the phænomena of capillary attraction as appear calculated to illustrate the subject.

(1). When a vertical cylinder or plate of glass is partly im-

* Had the stoppers been of platinum, what would have been the figure? 
mersed in water, a portion of the water rises immediately around them with a curved surface about $0 \cdot 1 \mathrm{in}$. above its na-

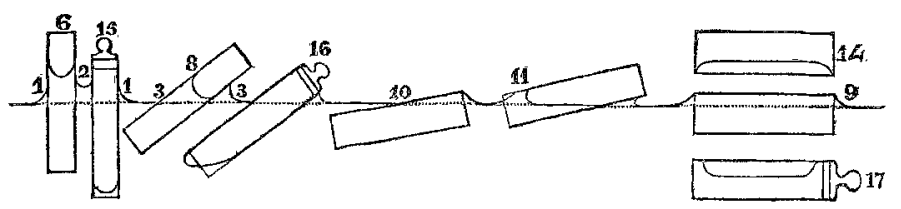

tural level. (2). If two vertical cylinders or two plates of glass be brought nearly in contact, the water will ascend higher between them than on the opposite or other sides. (3). On inclining a plate or cylinder of glass, the water under the overhanging side of either will rise to a greater perpendicular height than when they are vertical; but on the opposite side the elevation will be so much diminished as to be nearly insensible. (4). When two plates of glass, inclined to each other, are immersed in water with the line of their intersection vertical, the water will ascend between them, and form a hyperbola. (5). One end of a vertical tube having a bore of 0.5 inch being immersed in water, the water immediately surrounding its exterior appeared to stand as much above its natural level as within the tube. (6). But when capillary tubes, that is, those of which the bore did not exceed $0 \cdot 1$ inch, were made use of, the water within the tubes stood with a concave surface at a much greater height than on the outside; the discrepancy augmenting nearly as the bore of the tubes diminished. (7). Within a tube the ascent of the water, on account of the quantity of attracting zone being nearly double that of two vertical plates placed at a distance from each other equal to the diameter of the tube, is about double its elevation between the plates. (8). For the same reason the vertical height of the water within the half-inch tube when inclined, was greater on that interior side of it overhanging the water within, than on the corresponding exterior side (9). On drawing the same tube horizontally out of the water, it continued to be filled exclusively with that fluid, even when its interior upper surface was elevated nearly 0.1 inch above the general level. (10). This was equally the case when either end of the tube was submerged in, and the other stood out of the water not more than $0 \cdot 1$ inch. (11). On raising either end of the tube more than this height out of the water, the air began to intrude, impressing on the fluid the curved form exhibited in the figure. (12). The tube being subsequently 
held with the lower end rather more than $0 \cdot 1$ inch above the water, the air entered also at that depressed end, and in effecting a junction with the air previously introduced at the upper orifice, burst a film or thin plane of water extended across the tube almost close to the depressed end. (13). This film (which would abruptly form again, and in the same place on lowering the tube) was ruptured, on taking the tube horizontally out of the water, at the middle of the length of the tube. (14). When completely out of the water, a portion of that fluid remained at the bottom of the horizontal tube at a depth of about 0.1 inch. (15). One end of a tube, having a bore capable of raising water half an inch above its level, being hermetically closed, a disk of thin paper covering the other orifice was secured to the tube by means of melted wax. This end (purposely left rather moist within) being placed vertically in water at a considerable depth, the wax and paper were forced completely off; yet the water, from the resistance of the included air, did not rise more then $0 \cdot 1$ inch within the tube. (16.) Repeating the experiment with the half-inch tube, the water stood within it about the preceding height; but in bringing the tube nearly horizontal, the included air protruded a littie beyond the upper part of the orifice, continuing to escape gradually in small bubbles. (17.) When the tube, wholly immersed in the water, appeared to be exactly horizontal, the residue of the air, in figure like the bubble of a level, reached within 0:1 inch of each end, and did not attempt to escape until the stoppered end was slightly depressed, when the whole rushed abruptly out. But when the tube, in a subsequent experiment, contained only a small quantity of air, so that the (more spherical) bubble was at a considerable distance from each end, the stoppered end was obliged to be depressed several degrees, before the bubble could complete its escape.

On a careful review of the experiments, will not a diminished specific gravity of the water immediately in contact with the glass, together with the cohesion of its particles, account for every observed violation of hydrostatics? In a narrow tube the particles of water within it may be considered as equally acted upon by the maximum of the force pecnliar to the surface of the glass which tends to diminish their specific gravity; whereas the particles within a wide tube are mostly at some distance from that force, especially. where it is greatest, and are also influenced in a contrary sense by the cohesion of a greater number of particles beyond the effect of the force in question. Hence the same external pressure causes the lighter water within the small tube to rise higher than the heavier 
water within the wide one: and for the same reason the water fills the horizontal tube (9), even when held above its general level. No. 16 proves very clearly that a force of attraction perpendicular to the axis of the tube does not elevate the fluid, -or why should the included elastic air wholly prevent its wonted ascent? If such a force did exist, why should an almost insensible depression of the horizontal tube in No. 16, overcome it so completely as to expel the bubble of air? A globule of air rises to the surface of a liquid in consequence of the pressure upwards against its base being greater than the downward pressure on its upper surface : hence the greater the diameter of the globule, and the greater the force with which it is urged upwards; which may serve to account for the tardy and impeded escape of the minute bubble on repeating the 16th experiment. Besides, as the small bubble has to move at an almost insensible distance from the curved surm face of the glass, where the levity of the water must be very great, its specific gravity approaches more to that of the water in contact with its ends, and will therefore require a considerable depression of the tube to make the vertical column urging it below adequately heavier than the opposed one incumbent on it. But when the bubble is long and deep, the pressure upwards is derived from an incomparably longer and specifically heavier vertical column acting against diminished obtacles.

According to our theory, the specific gravity of the liquid within the horizontal tube of a level will be least at the upper corners of the tube; but along the upper surface, and still more so on the sides above the level of its axis, it will approach in a greater degree to its proper specific gravity. Hence the surface of sections of the liquid, whether perpendicular to, or in the direction of the axis, but especially the latter, will have a concave curve at the extremities; which will account for the peculiar figure of the bubble of a spirit-level.

(We may now comprehend how an uncurved tube containing a sufficient quantity of ether, \&c. becomes possessed of a bubble which does not extend to the ends of the tube. In a level of this description; as its bubble, when the cylindrical tabe is horizontal, will be stationary indifferently in any part of its length, and as the slightest inclination would displace the bubble (if sufficiently large) indefinitely, causing it to move at once to the elevated end of the tube, it could not serve to measure the minutest variation of inclination.)

Let the bubble of a curved tube pass over 0.1 inch for a variation of inclination of $1^{\prime \prime}$; then if its depth in the middle be 0.2 inch, its length, when the tube is horizontal, should be 
equal to 15 feet. But as the levity of the water near the extremities of the arc is greater than under the vertex, it must stand higher there than in the latter place, or an equilibrium cannot be effected. From this cause the length of the bubble becomes reduced from 15 feet to little more than as many tenths of an inch : however, as the reduction will be the same at each end, provided the tube be a perfect ring, the middle of the bubble will still coincide with the vertex of the arc. For reasons already assigned, it is nevertheless requisite that the bubble should be of a proper length and depth.

When a vertical plane describes any arc of revolution about a horizontal line or axis, a straight line, as the radius or chord of a circle, previously drawn anywhere on that plane, will have its inclination to the horizon varied by an angular quantity equal to that arc of revolution. This will be as obvious for an excentric line as it is for one intersecting the axis, when we consider that their parallelism or angle of inclination to each other remains constant. If we therefore describe with the same radius two circles on the vertical plane, one concentric and the other excentric to its axis, and mark their vertex points before, and also after any partial revolution of the plane, the lineal distance of the two points on the one circle will be exactly equal to that of the corresponding points on the other. Were we to fix anywhere on (but parallel to) either vertical side of our circular vessel ${ }^{*}$ the tube of a spirit-level having an equal radius of curvature, then, as the vessel revolved, its bubble would pass over the same lineal space as that of the tube. Should the radius of curvature of the tube exceed that of the vessel, the arc of revolution (or variation of inclination), as measured by the graduations on the rim of the latter, would nevertheless correspond with the indications of the scale of the level. With this explanation we may now comprehend how a spirit-level, having a radius of curvature of several hundred feet, although fixed (parallel) to a vertical plane (such as that of an astronomical circle) within a few inches of the axis of rotation, should have its bubble displaced by the same (sensibly) lineal space as though that axis coincided with the centre of its circular curve.

To construct an instrument called a level, capable of determining the horizontal inclination of straight lines, planes, \&c. the curved tube of a spirit-level furnished with a scale is fixed with its convex side upwards to the upper surface of a straight bar of brass, wood, \&c. in such a manner that the plane of the circle of curvature of the tube shall be perpendicular to

* See abore, page 257. 
the under surface (a right-angled parallelogram) and paralle to the (longitudinal) sides of the bar or frame*.

If we mark the vertex (or most elevated point) of the arc of any segment of a circle previous to its revolution about an inscribed vertical line touching the arc in any point, then will the true vertex and the initial mark coincide during the revolution; and the chord of the (vertical) segment (or a plane cutting it at right angles), if perpendicular to the vertical line, will continue horizontal throughout, and describe a perfectly horizontal plane. Now as the middle point of the bubble of a spirit-level will always come to rest at the vertex of the circular arch of its tube, represented by the arc of the segment; and as the intersection (at right angles) of the under surface of the level by the vertical plane of the circle of curvature is equivalent to the chord of the segment, (and that under surface to the plane cutting it at right angles, ) we may be certair that the surface on which the level may be moved about in exact contact, without displacing the bubble, is a plane parallel to the horizon. Or, as the ends of the bubble do not de-viate from their marks on the tube, or divisionis on the scale, the under surface of the level preserves during the revolution its parallelism to the surface of the liquid (or base of the bubble), which is always horizontal, and must therefore move. parallel to a horizontal plane.

When the level rests on a horizontal plane, with the ends of the bubble coinciding with the two marks drawn on the tube, or with each end at the same distance from the zero of the scale, it is said to be adjusted; in which case the middle of the bubble is at the point of bisection of that arc of the circle

* If the circle $\mathrm{C}$ move with its centre on the circumference of the larger circle $B$, its plane being always perpendicular to that of the latter, and in the direction of the line joining their centres, then will its circumference generate a ring similar to the curved tube. The circle described by that point $v$, of the circumference of the smaller circle $\mathrm{C}$, which lies in the direction of the plane of the great circle $B$ (and of the straight line connecting their centres) will therefore represent the circle of curvature of the tube.

Having placed the tube with its axis

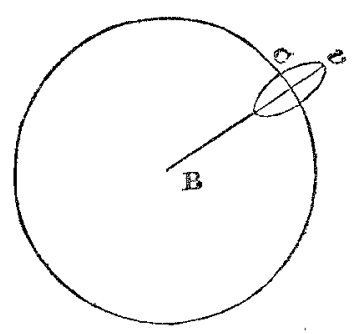
parallel to the sides. of the frame, bring the mark indicating the situation of some point of the circle of curvature to its greatest elevation above the under surface of the frame, when it will lie in the plane passing through the axis of the tube perpendicular to that under surface. 
of curvature, of which the line of intersection of its plane by the (horizontal) under surface of the level is the chord.

As the sides of the level are perpendicular to its under surface, if we fix a short tube to the trame at right angles to the principal one, and mark the ends of its bubble when the level rests on a plane found to be horizontal, we shall know in future that the sides of the frame, and consequently the parallel plane of the circle of curvature, will be vertical when the bubble of the transverse tube comes to rest between its marks.

It is also evident, that if we place the level with either of its (perpendicular) sides in contact with a vertical plane so that its under edge (or corresponding longitudinal line of the under surface) lies exactly on, parallel to, or in the direction of a straight line described on that plane, and find on reversing the level (that is, on making the opposite side of the level to press against the vertical plane with its under edge coinciding with the straight line) that the ends of the bubble come to rest at the same marks, it proves that the line is horizontal.

If a segment of a circle be made to revolve about an inscribed line inclined to the horizon, and touching its arc in any point, its chord, if perpendicular to that line, will describe an inclined plane. A horizontal line being drawn on this plane through the point in which the line of revolution, if produced, would touch it, another straight line lying in the same plane and passing through that point at the greatest possible angular inclination to the horizon, also that of the plane, will intersect that horizontal line at right angles*. Then, if we mark the vertex of the arc when the segment is vertical, that is when in the direction of the inclined line drawn on the generated plane, the mark will attain its maximum distance from the true vertex, varying as the segment revolves, at the completion of a semi-revolution; at which period the reversed segment becomes vertical again, and coincides with the inclined line for the second time; and half this maximum distance, as measured on the graduated arc of the segment, will be equal to the zenith distance of the line of revolution, and to the horizontal inclination of the plane and generating chord. At onefourth, and again at three-fourths of the revolution, the plane of the segment will be at its greatest inclination to the horizon,

* The intersection of an inclined plane through any point on it by a horizontal plane, is a straight line parallel to the horizon; and the intersection of the same plane by a vertical one passing through any point of it perpendicular to its horizontal (parallel) lines, will be a straight line having in common with lines parallel to it, an inclination to the horizon greater than that of any other line that can be drawn on the same plane.

Nere Series. Vol. 1. No. 5. May 1827. $3 \mathrm{~A}$ equal 
equal to that of the inclined plane, when the chord, then in the direction of the horizontal line described on the inclined plane, will be horizontal, and the vertex be situated at the point of bisection of the total arc, and of that portion of it comprehended between the initial mark and the vertex at its greatest elongation from it. Or conceive an adjusted level to be placed so as to coincide in the first instance with any horizontal line drawn on the inclined plane, and subsequently with one at right angles to it, or in the direction of the line on which the inclination of the plane is measured; and it will be evident that the bubble must have advanced from zero towards the elevated end of the level, by a distance on the scale equal to the inclination of the plane. But on reversing the level, the same end, (repassing the horizontal line with the bubble re-verted to zero, will be depressed equal to its previous elevation, and the bubble must consequently come to rest at the same distance from zero as before, but on that side of it the nearest to the other (now elevated). end of the level; so that the arc of distance passed over by the bubble will equal troice the inclination of the plane.

To find the angular inclination of a plane, move the level about on its surface until the bubble has made its maximum approach to the (elevated) end of the tube. Having registered the divisions on the scale giving the middle point of the bubble, proceed with the rotatory movement of the level until its direction is reversed, which will occur when the bubble has made its greatest approach to the other end of the tube, and ascertain the middle point of the bubble as before. Half the sum of these two middle points when the signs are unlike, or half their difference when the signs are like, will be equal to the angle of inclination of the plane*. When the level is placed on the inclined plane in such a direction that the bubble settles at that point of the scale answering to the half-difference of these two middle points, the longitudinal lines of the under. surface of the level are horizontal, and its corresponding sides inclined at an angle equal to that of the planet.

- When the level is furnished with a transverse tube properly adjusted, we are enabled to place it at once on the inclined plane, in the direction of its greatest inclination, which will be, when the sides of the level are vertical, known by the bubble of the transverse tube coming to rest between its marks. + On this account the bubble would deviate (at right angles) from the equally inclined circle of curvature, and horizontal lines cannot be safely. drawn by a similar Ievel on the inclined plane with which its under surface is in contact. To draw them correctly, the sides of the level must be preserved vertical by means of the transverse tube, whilst one of the longitudinal lines of the under surface rests on the inclined plane with the bubble of the principal tube at the proper divisions. 
The measurement of the angular inclination of a line lying in a vertical plane does not differ essentially from that of a line of maximum inclination described on an inclined plane, but is susceptible of more evident demonstration. Let $A B$ and $\mathrm{CD}$ be two straight lines lying in the same vertical plane

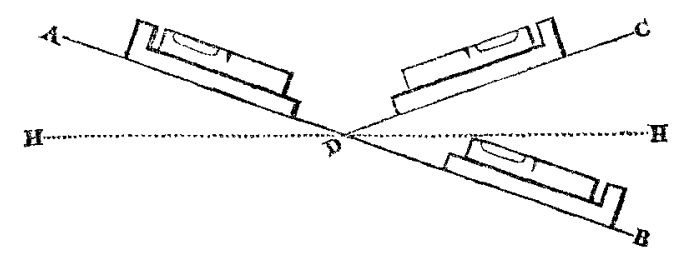

and equally inclined to the horizontal line HH. Place the under edge of either (vertical) side of a level on $A B$, and, having marked the ends of the bubble, reverse the level, and make the (opposite) under edge coincide with DC; when the bubble, as the same end of the level is equally elevated as before, will come to rest at the initial marks. Depress this elevated end until the under edge coincides with $A B$, when the bubble must have passed over a distance on the scale equal to the angle CDB, evidently the sum of the two equal angles $\mathrm{CDH}$ and $\mathrm{HDB}$, either of which is equal to the inclination of the line $A B$ (or that of $C D$ ). In depressing the reversed level from its position on $\mathrm{DC}$ to that on $\mathrm{AB}$, its under edge must have coincided with, or been parallel to the horizontal line $\mathrm{HH}$ when the bubble had run over half the distance answering to the double inclination of $\mathrm{AB}$, equal angle $\mathrm{CDB}$. Hence the under surface of the level will be horizontal when (its sides being vertical) the middle of the bubble settles at a point on the tube, or division on the scale corresponding to the point of bisection of the space run over by the bubble, on reversing the level on any inclined line. When this point falls exactly between the two marks drawn on the tube, or on the zero of the scale, the level is properly adjusted. When this is not the case, turn the adjusting screws until the bubble settles on the elevated side of zero at a distance from it equal to the inclination of the line on which the level rests. Should the error of adjustment be trivial, it is more advisable to ascertain and register its value every time the level is made use of, than to attempt to correct it by the screws; for variations of temperature not only cause the vertex of the tube to alter its position $3 \mathrm{~A} 2$ relatively 
relatively to the under surface of the level *, but have also the effect of disturbing for some time the adjusting screws, and probably other parts of the mounting. On measuring by an adjusted level a number of planes or lines differing in inclination, the half-difference of the space passed over by the bubble should always be zero, or the instrument cannot be considered as perfect.

Leeds, March 5, 1827.

ErratuM.--Page 260; for 200,000, read 206265; (the number by which the length of a division on the scale of a level answering to $l^{\prime \prime}$ must be multiplied to obtain the length of the radius of its curvature).

LXXII. A Synopsis of the Birds discovered in Mexico by W. Bullock, F.L.S. and H.S., and Mr. William Bullock, jun. By William Swainson, Esq. F.R.S. F.L.S. \&c.†

THE intercourse which recent political events have opened 1 between Mexico and Great Britain, promises to be no less interesting to zoological science, than important to the commercial prosperity of both nations. Mr. Bullock was among the first of our countrymen, whose ardent curiosity led him to visit those distant shores; and the scientific treasures with which he returned, bear ample testimony to that zeal and indefatigable indistry which has ever marked his pursuits. The exhibition of these objects, together with the valuable models and relics of Mexican antiquity, which this enterprising traveller procured during the short space of his sojourn, attracted the public attention for two years. That such a collection, invaluable to the historian as throwing a new light upon the ancient state of one of the most extraordinary nations of antiquity, should have been suffered, in these days, to have been dispersed by the hammer of the auctioneer, will excite the deep regret of every friend to knowledge. They may now, indeed, serve as objects of mere curiosity, but those advantages which the historian and the antiquary might have derived from their study and investigation, as a whole, are for ever lost.

The zoological suljects, possessed by no museum in Europe, shared the same fate, but not before Mr. Bullock had placed

* When the temperature increases, the vertex removes to a point of the tube nearer the thicker end. In some levels now in my possession the variation is 1 " for every 2 degrees of Fahrenlueit's thermometer.

+ Communicated by the Alithor. 